\title{
Analysis of Signalling Time of Community Model
}

\author{
Boudhayan Bhattacharya ${ }^{\mathrm{a}, *}$, Banani Saha ${ }^{\mathrm{b}}$ \\ ${ }^{a}$ Sabita Devi Education Trust - Brainware Group of Institutions, 398 Ramakrishnapur Road, Barasat, Kolkata \\ -700124, West Bengal, India \\ ${ }^{b}$ University of Calcutta, JD-2, JD Block, Sector III, Bidhannagar, Kolkata - 700098, \\ West Bengal, India
}

\begin{abstract}
Data fusion is generally defined as the application of methods that combines data from multiple sources and collect information in order to get conclusions. This paper analyzes the signalling time of different data fusion filter models available in the literature with the new community model. The signalling time is calculated based on the data transmission time and processing delay. These parameters reduce the signalling burden on master fusion filter and improves throughput. A comparison of signalling time of the existing data fusion models along with the new community model has also been presented in this paper. The results show that our community model incurs improvement with respect to the existing models in terms of signalling time.
\end{abstract}

Index Terms: Master Fusion Filter; Reference Sensor; Local Filter; Community Model; Data Transmission Time; Normalized Signalling Time.

(C) 2015 Published by MECS Publisher. Selection and/or peer review under responsibility of the Research Association of Modern Education and Computer Science

\section{Introduction}

Data fusion is a state-estimation process based on data from multiple systems or data sources. As numerous information sources are available via inexpensive network connections, the amount of data available in repository are increasing day by day. These data are collected and often sorted to get the desired decision for a particular problem. The data fusion methodology is often used to access all these sources through a consistent interface to conclude a particular desired decision. The information integration system is used to obtain a complete yet concise overview of all existing data without accessing all data sources separately. The data fusion methodologies are used in different areas like maintenance engineering, robotics, pattern recognition and radar tracking, mine detection and other military applications, remote sensing, traffic control, aerospace systems, law enforcement, medicine, finance, meteorology and geo-science. The literature available till date,

* Boudhayan Bhattacharya Tel.: +91-33-7144-5608; fax: +91-33-2584-7273

E-mail address: mailforboudhayan@gmail.com 
discusses various models and naturally the models are of prime importance as the structure defines the working principle in terms of the practical implication. It was found that all the proposed models try to decide the working principle based on a particular practical scenario. The research work published to date does not provide any systematic approach towards calculating the signaling time between different data fusion filter levels, based on different data fusion methodologies. In this paper, the signaling time of community model [1][2] has been calculated and compared with the existing models like Waterfall Model, Multisensor Integration Fusion Model, Behavioural Knowledge-based Data Fusion Model, Omnibus Model, Dasarathy Model and JDL Model[10]-[14] and the results show that the community model requires less signaling time due to the verification with reference sensor in each stage. This paper is organized as follows -Section 2 deals with the classical data fusion models and their contributions. Section 3 introduces the Community Model and deduces formula to calculate the signaling time based on the model. The corresponding algorithm for calculation of the signaling time has also been discussed here. Section 4 deals with the performance analysis of the new model along with the other existing models in terms of signaling time.

\section{Nomenclature}

\begin{tabular}{|ll|}
\hline $\mathrm{P}_{\mathrm{n}}$ & Perimeter of a data fusion filter \\
$\mathrm{F}$ & Data fusion density per local filter \\
$\mathrm{S}$ & Flattening of the local filter surface \\
$\mathrm{A}$ & Scale factor stability \\
$\mathrm{N}_{\mathrm{R}}$ & Alignment \\
$\mathrm{H}$ & Random noise \\
$\mathrm{V}$ & Bias uncertainty \\
$\mathrm{N}$ & Average velocity of the light \\
$\mathrm{D}$ & Total number of local filters \\
$\delta$ & Data for master fusion filter \\
1 & Filter Perimeter \\
$\mathrm{n}$ & No. of cells in the filtering area \\
$\alpha$ & Ratio of active filter to total filters \\
$\lambda_{\mathrm{a}}$ & Incoming data session rate \\
$\lambda_{\mathrm{d}}$ & Outgoing data session rate \\
$\mathrm{d}_{\mathrm{L} 1 \mathrm{~L} 2}$ & Average distance between two filter levels \\
$\mathrm{R}_{\mathrm{c}}$ & Filter crossing rate \\
$\mathrm{R}_{\mathrm{w}}$ & Hop weight ratio \\
$\mathrm{R}_{\mathrm{l}}$ & Average filter registration signalling refreshing rate \\
$\mathrm{L}_{\mathrm{T}}$ & Latency Time \\
$\mathrm{T}_{\mathrm{R}}$ & Data transmission time \\
$\mathrm{C}_{\mathrm{n}}$ & Normalized Signalling Cost \\
$\mathrm{C}_{\mathrm{S}}$ & Signalling Cost \\
$\mathrm{C}_{\mathrm{T}}$ & Cumulative Signalling Cost \\
$\mathrm{P}_{\mathrm{T}}$ & Processing Delay \\
$\mathrm{S}_{\mathrm{T}}$ & Signalling Time \\
\hline
\end{tabular}

\section{Related Work}

The discussions within the GDR-PRCISIS [3] work group on information fusion, formulated the basic architectural specifications for data fusion. European work group FUSION, worked on fusion from various perspectives in 1996 to 1999 [4]. The Multi-Sensor Data Fusion (MSDF) was introduced for military 
applications [5][6] and then are applied to civil industries[7]-[9]. The popular filter models available in literature are: Waterfall Model, Multisensor Integration Fusion Model, Behavioural Knowledge-based Data Fusion Model, Omnibus Model, Dasarathy Model and JDL Model [10] - [14]. The Waterfall Model and Multisensor Integration Fusion Model take the data from all navigation sensors [10]-[12]. Behavioural Knowledge-based Data Fusion Model uses outputs of one filter as inputs to a subsequent filter [10]. The Omnibus Model and Dasarathy Model are two-stage filtering models where all parallel local filters combine their own sensor systems with a common reference system [10]-[14]. The JDL Model is a generic model which combines two approaches of data fusion - Measurement Fusion and State Fusion[10][11]. The signaling time parameter is used to measure the transmission of a signal. They are used in all the areas where data transmission is required to analyze or fuse data [15][16]. This paper provides a scheme for the calculation of the signaling time of the community model.

\subsection{Waterfall Model [10]}

Harris founded the waterfall model [10]. The figure below represents the model. From the figure it is observed that data flow operates from the data level to the decision making level. The continuous updation of sensor system is carried out on a steady basis with feedback information that arrives from the decision-making module. The feedback element provides advice to the multi-sensor system on re-configuration and data gathering aspects etc. The three levels of representation in the waterfall model are:

Level 1: Transformation of raw data is carried out to provide the requisite information about the environment. For this to be achieved, the models of sensors are required. The models could be based on either physical laws or experimental analysis.

Level 2: This level comprises of feature extraction and thereafter the fusion of those features. This is done to obtain a symbolic level of inference about the data. The main objective here is to minimize the data content and maximize the amount of information delivered thereby. The output of this particular level is a list of estimates with the corresponding probabilities.

Level 3: This level maps objects to the corresponding events. On the basis of information that has been gathered, the available databases and libraries, and the human interaction, the possible routes of action are assembled.

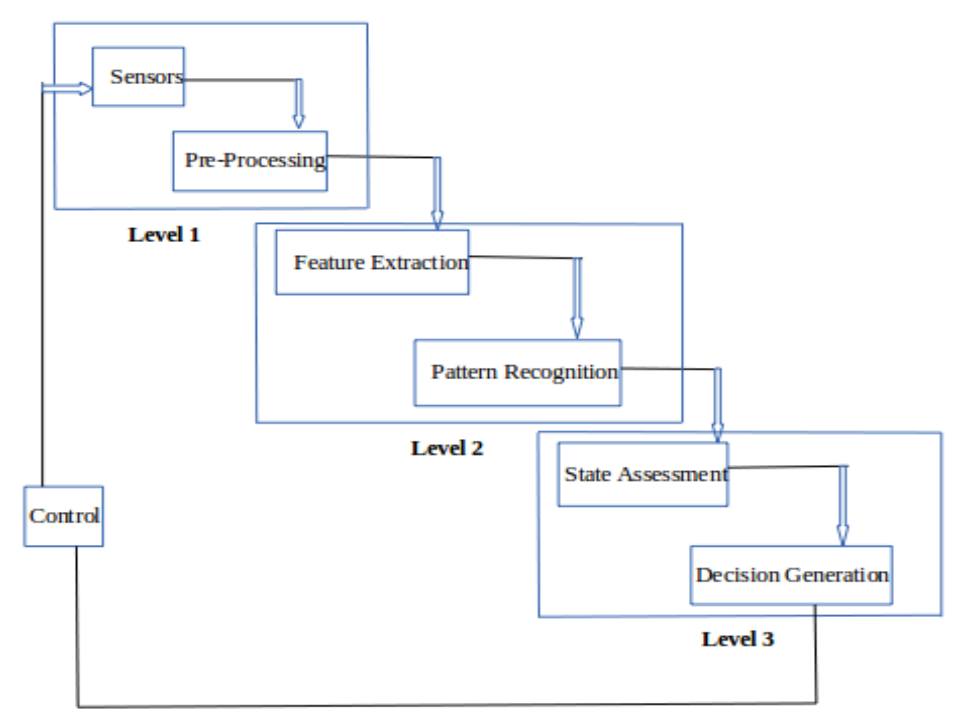

Fig. 1. Waterfall model [1] 


\subsection{Multi-sensor Integration Fusion Model [11][12]}

Luo and Kay brought to light a generic data fusion structure which was based on multi-sensor integration [11][12]. Here, data from the different sources was collected and combined in a hierarchical manner within the embedded fusion centres. Using this architecture a clear distinction was made between multi-sensor integration and multi-sensor fusion.

Multi-sensor integration is all about the usage of multiple sensor information in order to assist in a particular task, whereas multi-sensor fusion refers to any arbitrary stage of the integration process where the actual combination of data takes place.

From the figure describing the architecture, it is evident that data is collected at the sensor level which is then transferred to the fusion centres. The fusion process is thereafter carried out in the fusion centres which in turn could be done in a hierarchical and sequential manner. An information system is incorporated in the fusion process and is highly useful in the sense that it contains the relevant databases and libraries.

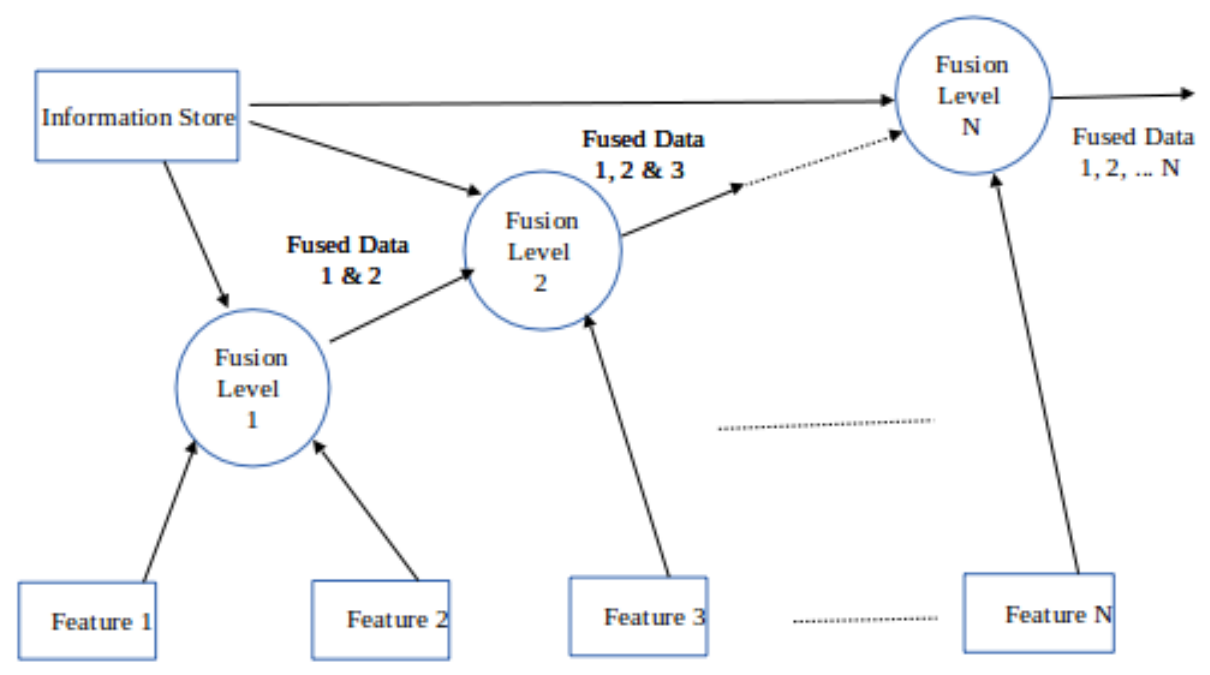

Fig. 2. Multi-sensor Integration Fusion Model

When the combination of information takes place at the different fusion centres, the representation level also increases from signal level to symbol level.

Table 1. Characteristics of data fusion levels

\begin{tabular}{lllll}
\hline Characteristics & Signal level & Pixel level & Feature level & Symbol level \\
\hline $\begin{array}{l}\text { Representation level of information } \\
\text { Types of sensory information }\end{array}$ & Low & Low & Medium & High \\
& $\begin{array}{l}\text { Multi- } \\
\text { dimensional }\end{array}$ & Multiple images & Features & $\begin{array}{l}\text { Decision logic } \\
\text { from signals / } \\
\text { signal }\end{array}$ \\
Models of sensory information from & $\begin{array}{l}\text { Random variable } \\
\text { sith noise }\end{array}$ & $\begin{array}{l}\text { Random / images } \\
\text { across the pixel }\end{array}$ & $\begin{array}{l}\text { Non-invariant } \\
\text { form of features }\end{array}$ & $\begin{array}{l}\text { Symbol with } \\
\text { degree of } \\
\text { uncertainty }\end{array}$ \\
\hline
\end{tabular}

\subsection{Behavioural Knowledge-based Data Fusion Model}


Pau has described a data fusion framework based on behavioural knowledge. From the figure it is clear that this framework comprises of a few basic stages which necessarily needs to be computed before the output is established.

According to this framework, firstly a feature vector is extracted from the raw data. The feature vector is associated to the defined features, thereafter fusion is carried out at the data analysis levels and at the sensor attribute. Finally, a set of behavioural rules are extracted as a final representation of the fused output. This model describes the following three levels of representation:

(i) In the lowest level, corresponding to each sensor, there exist a vector space with measured parameters and coordinate dimensions.

(ii) The second level attaches labels to the appropriate or relevant features that are in turn extracted from these vectors.

(iii) Lastly, the third level comprises of a set of formalisms which relates or maps the feature vectors to the events.

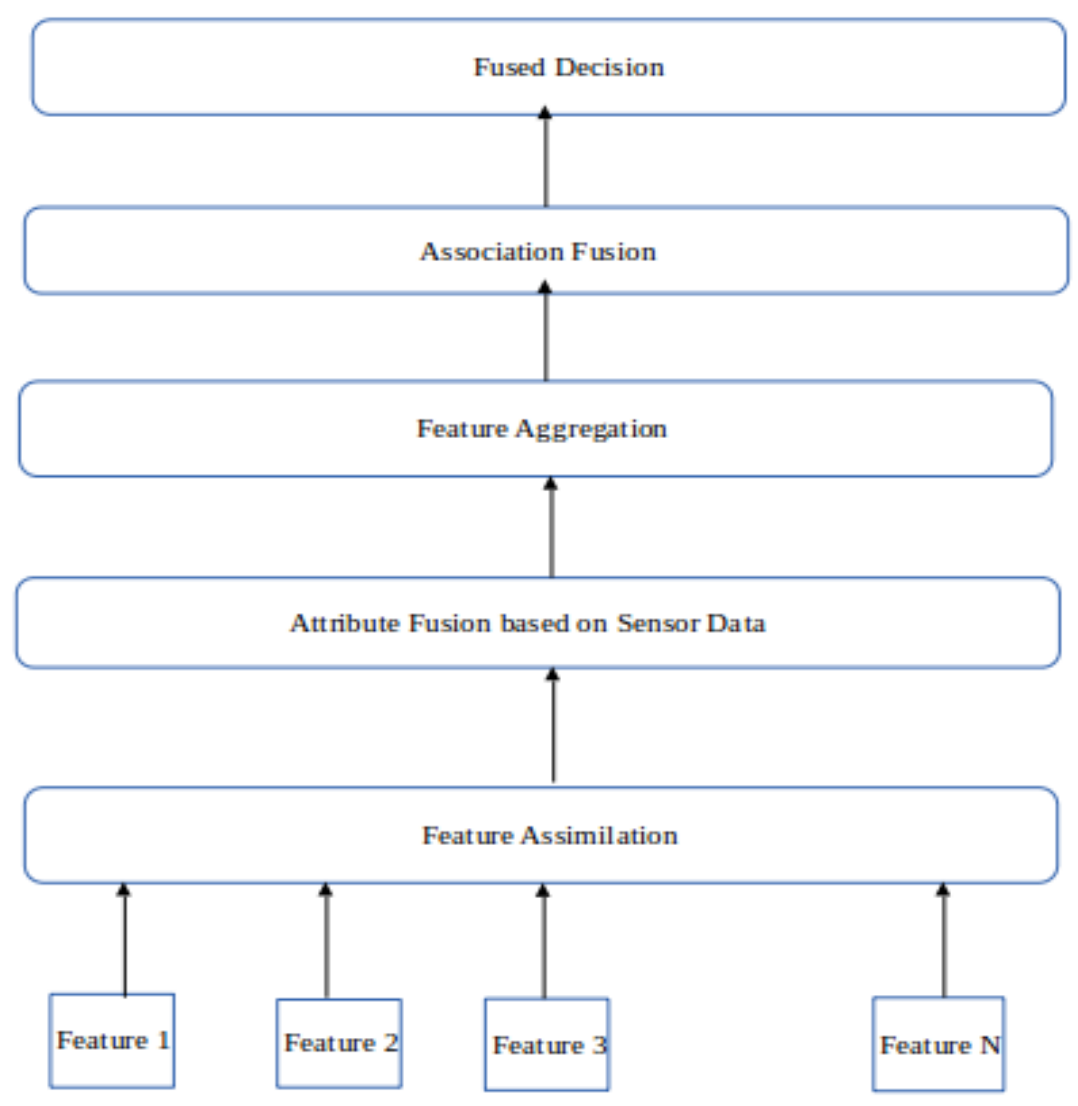

Fig. 3. Behavioural Knowledge-based Data Fusion Model

\subsection{Dasarathy Model}

According to many researchers, the three main levels of abstraction during the data fusion process are - Data, Feature and Decision. 
Data-This is precisely the sensor data.

Decisions -This includes symbols or belief values.

Features -These are the intermediate-level information.

Dasarathy pointed out however that fusion might occur both within these levels and as a means of transformations between them.

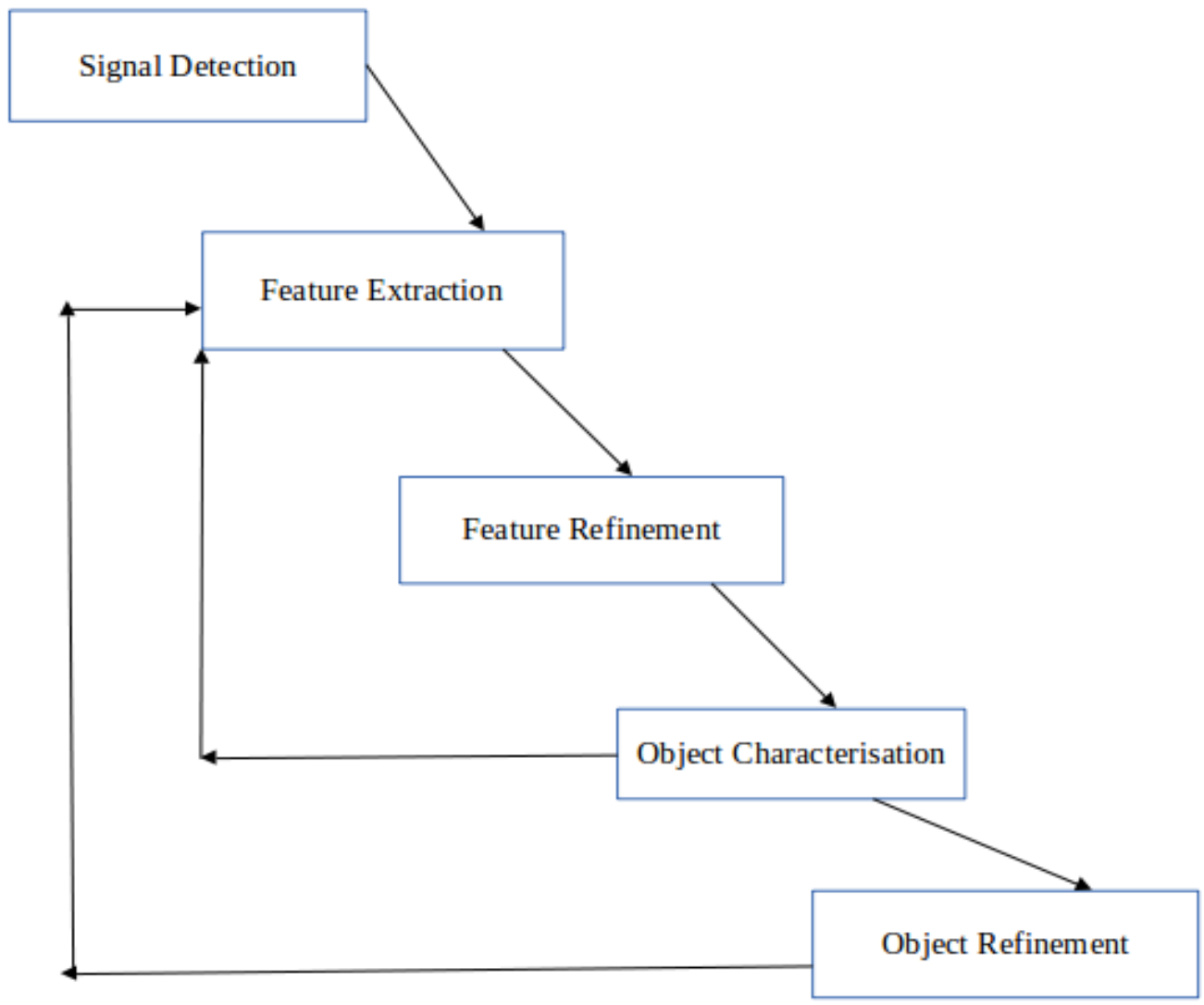

Fig. 4. Dasarathy Model

\subsection{Omnibus Data Fusion Model}

This process model, introduced by Bedworth and O'Brien is a hybrid of the three models namely Boyd loop, Dasarathy, and Waterfall models. The figure below describes the four main modules of this framework. These models address the variety of tasks in data fusion and its functional objectives. The Boyd loop is an iterative process with four elements namely observe, orient, decide and act, which operates in a closed loop. 


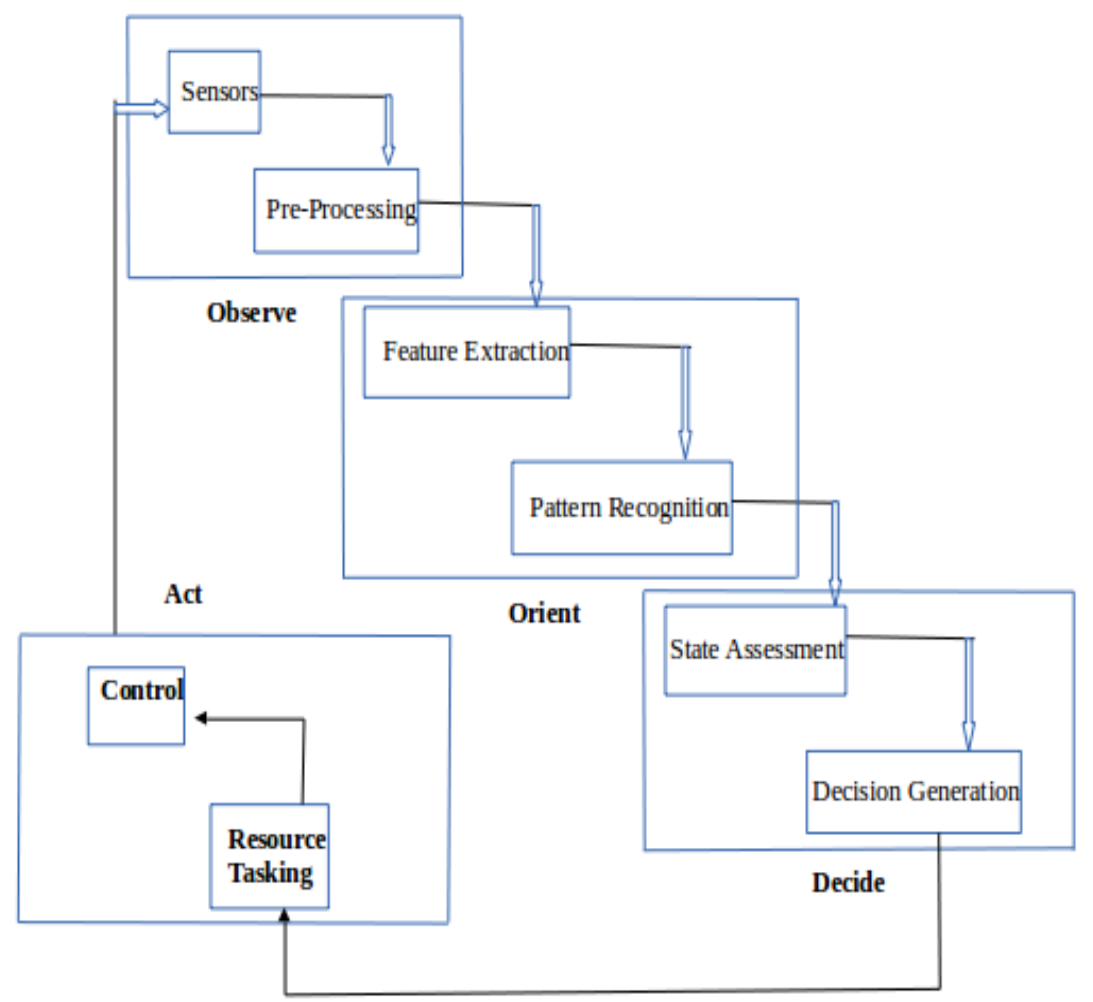

Fig. 5. Omnibus Data Fusion Model

\subsection{JDL Model}

The most famous of the data fusion model is the Joint Directors of Laboratories (JDL) model. The JDL model was created by the Data Fusion Working Group which was established in 1986 with the objective of unifying research terminology in order to promote technology transfer and cooperation between the groups.

As per this model, the information sources which are used for data fusion can very well include both local and distributed sensors(ones that are physically linked to the other platforms), or environmental data, a priori data, and human guidance or inferences. According to Llinas and Hall, the JDL data fusion process contains the six different levels of processing as described below:

Level 0 Processing (Source Pre-processing): This is used for rearrangement of the data which is collected from different sensors within a common time as well as space reference system (data alignment) for the removal of redundant information which is acquired by the different sensors or to filter out the wideband noise as well. This level involves association and characterization of sensed signals. Associating signals means assigning them to the same entity e.g., tracked target) of the environment. Activities like pixel/signal processing are also involved here however no semantic meaning is assigned to the assessed data here.

Level 1 Processing (Object Refinement): In this level (also called data fusion or multisensor integration) combination of data is carried out to assign dynamic features (e.g., velocity) as well as static (e.g., identity) to objects, hence adding semantic labels to data. Techniques used in this level include data association and management of objects. The study of grounding problem in the artificial intelligence community is related to this level.

The three main objectives of this level are: 
(i) Performing a correct association between multiple entities and the sensor data.

(ii) Estimation of those parameters or attributes which are most significant for the application that is considered.

(iii) Identification of an entity based on a set of extracted features.

Level 2 Processing (Situation Refinement): This level involves elaboration of the output results that are obtained from level 1 processing in order to extract useful requisite information about the relationships between multiple entities which are located in the same environment i.e. it involves aggregation of Level 1 entities into high-level, more abstract entities, and relations between entities. Here assessment of input data is done with respect to the environment, relationship among Level 1 entities, and entity patterns in space and time.

Level 3 Processing (Threat Refinement): Level 3 is also called significance estimation or impact assessment. This level enables us to make future predictions on the basis of current situation for the detection of possible threats if any. It estimates and predicts the combined effects of system control plans and the entities of Level 2. This level includes estimation and prediction of effects on situations of planned or estimated/predicted actions by the participants including interactions between action plans of multiple players (e.g. assessing susceptibilities and vulnerabilities to estimated/predicted threat actions given one's own planned actions).

Level 4 Processing (Process Refinement/Resource Management): Level 4 takes up the responsibility of monitoring and controlling the data fusion process on a wholesome basis in order to assess and also to improve its real-time performance based on possible application-specific needs or operational requirements. This level evaluates the performance of the data fusion process and includes everything that refines it. e.g., acquisition of more relevant data, selection of more suitable fusion algorithms, optimization of resource usage with respect to, for instance, electrical power consumption.

Level 5 (Fused Data): Level 5 fuses the refined data derived from the level 4. The fusion of data is based on the content and context so that a formal decision can be taken on the basis of those processed data.

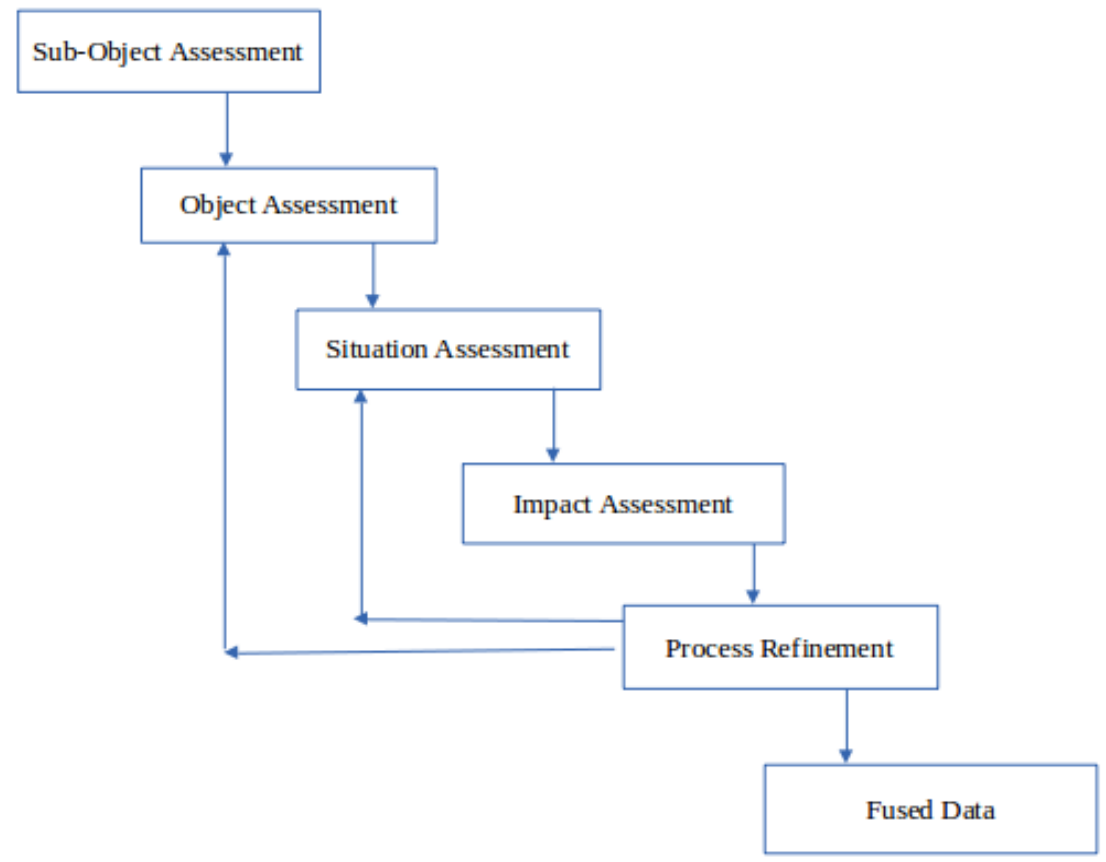

Fig. 6. JDL Model 


\section{Signaling Time Estimation of Community Model}

Data fusion models are based on either parity vector/space techniques [17]-[19] or comparison method [20][22]. Using any of these techniques, the minimum and maximum residual magnitude can be measured [23]. These methods compute and compare each parity equation and deploy a least-squares estimator for the estimation of the measured states. Naturally it is a time-expensive procedure for a large number of redundant sensors.

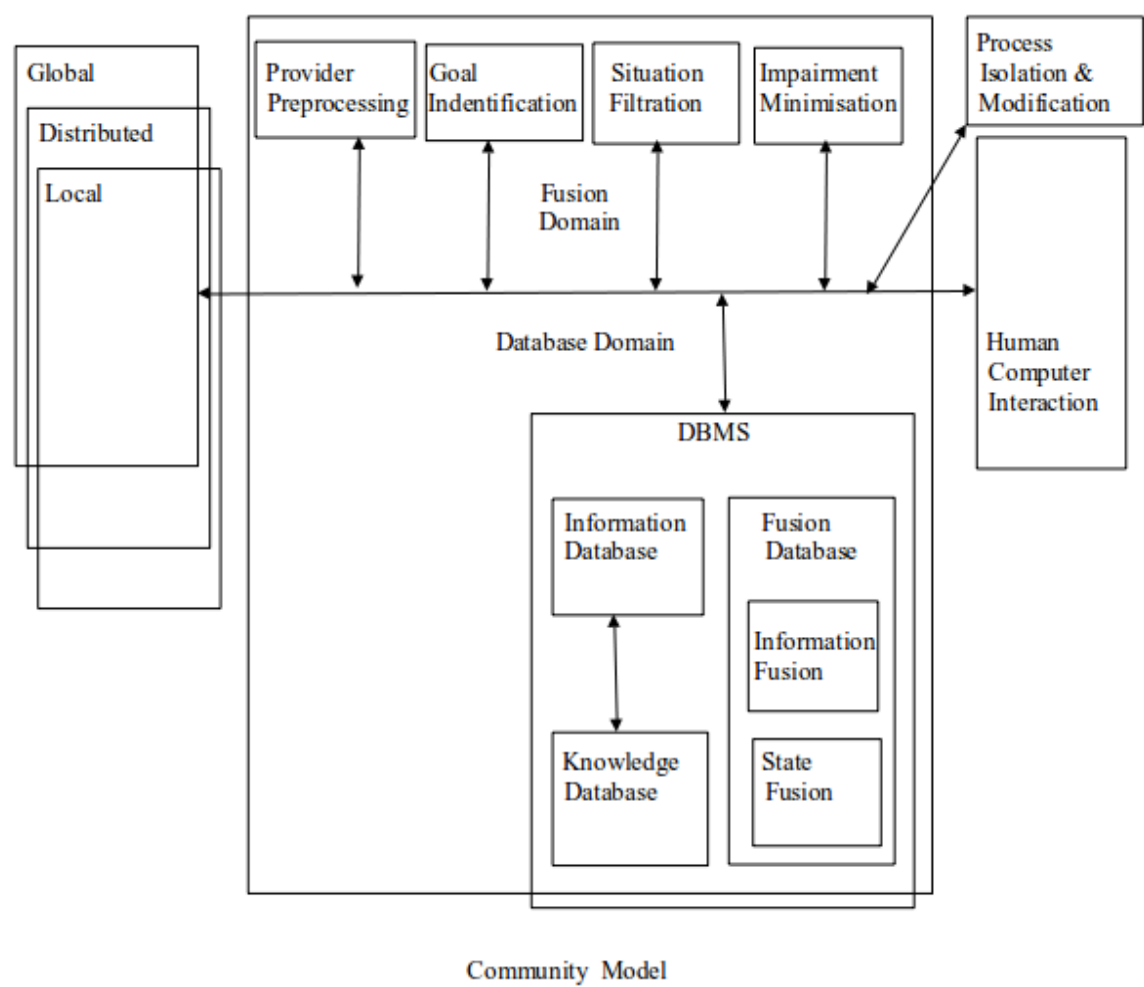

Fig. 7. Community Model

\subsection{Mathematical formulation for Signaling Time}

Here the mathematical formulation of the community model is deduced to calculate the signalling cost for the data fusion using the Dynamic Transformation Model.

The general n-level LF transmission time for the single master fusion filter domain [1] is :-

$$
\mathrm{T}_{\mathrm{R}}=\sum_{1}^{N} \mathrm{~L}_{\mathrm{T}}^{*} 1 / \mathrm{A}\left[\mathrm{S}\left(\mathrm{H}^{*} \rho \mathrm{vP}_{\mathrm{n}} / \pi\right) / \mathrm{F}+\mathrm{N}^{*} \mathrm{~N}_{\mathrm{R}}\right]
$$

Where

A = Alignment,

$\mathrm{S}=$ Scale factor stability, 
$\mathrm{H}=$ Bias uncertainty,

$\rho=$ Data fusion density per LF,

$\mathrm{v}=$ Average velocity of the light,

$\mathrm{P}_{\mathrm{n}}=$ Perimeter of each LF,

$\mathrm{F}=$ Flattening of the LF surface,

$\mathrm{N}=$ Total number of $\mathrm{LF}$,

$\mathrm{N}_{\mathrm{R}}=$ Random noise

The signalling time is a factor based on data transmission time and the processing delay involved in each of the filter level pairs. The processing delay for each pair for $n$ number of levels denoted by $\mathrm{P}_{\mathrm{T}}$ is -

$$
\sum_{\mathrm{PT}=\mathrm{N}}^{N} \sum_{\mathrm{Cn} *}^{N}{ }_{1} \mathrm{Cs}
$$

So the signalling time $\left(\mathrm{P}_{\mathrm{T}}\right)$ for $\mathrm{n}$ levels of filter is -

$$
\mathrm{ST}=\mathrm{TR} * \mathrm{PT}
$$

\subsection{Algorithm for Signaling Time}

The signalling time is estimated based on the data transmission time and processing delay based on the two immediate filter levels. First the data transmission time is calculated. After that the processing delay for $\mathrm{n}$ level of filters is calculated. From equation (3) it is evident that signalling time is the product of data transmission time and processing delay. The algorithm has been designed based on the previous work in [1][2][25].

\section{Procedure: Signalling Time}

1. Begin

2. input $\mathrm{RS}_{\mathrm{A}}, \mathrm{RS}_{\mathrm{S}}, \mathrm{RS}_{\mathrm{H}}, \mathrm{RS}_{\rho}, \mathrm{RS}_{\mathrm{V}}, \mathrm{RS}_{\mathrm{p}}, \mathrm{RS}_{\mathrm{N}}$,

3. set $\mathrm{T}_{\mathrm{R}} \leftarrow 0, \mathrm{C}_{\mathrm{n}} \leftarrow 0$

4. input $\mathrm{L}_{\mathrm{T}}, \mathrm{A}, \mathrm{S}, \mathrm{H}, \rho, \mathrm{V}, \mathrm{P}_{\mathrm{n}}, \mathrm{P}, \mathrm{N}, \mathrm{N}_{\mathrm{R}}, \mathrm{C}_{\mathrm{n}}, \mathrm{v}, 1, \mathrm{n}, \alpha, \lambda_{\mathrm{d}}, \lambda_{\mathrm{a}}, \mathrm{d}_{\mathrm{L} 1 \mathrm{~L} 2}, \mathrm{R}_{\mathrm{c}}, \mathrm{R}_{\mathrm{w}}, \mathrm{R}_{1}$

5. call IM

6. if $(\delta !=\varnothing) \| \mathrm{D} !=\mathrm{RS}$

7. $\quad$ update $\mathrm{A} \leftarrow \mathrm{RS}_{\mathrm{A}}, \mathrm{S} \leftarrow \mathrm{RS}_{\mathrm{S}}, \mathrm{H} \leftarrow \mathrm{RS}_{\mathrm{H}}, \rho \leftarrow \mathrm{RS}_{\rho}, \mathrm{V} \leftarrow \mathrm{RS}_{\mathrm{V}}, \mathrm{P} \leftarrow \mathrm{RS}_{\mathrm{p}}, \mathrm{N} \leftarrow \mathrm{RS}_{\mathrm{N}}$

8. endif

9. $\mathrm{i} \leftarrow 1$

10. while $(\mathrm{i}<=\mathrm{N})$

11. $\mathrm{T}_{\mathrm{R}}=\mathrm{T}_{\mathrm{R}}+\mathrm{L}_{\mathrm{T}} * 1 / \mathrm{A}\left[\mathrm{S}\left(\mathrm{H}^{*} \rho v \mathrm{P}_{\mathrm{n}} / \pi\right) / \mathrm{F}+\mathrm{N}^{*} \mathrm{~N}_{\mathrm{R}}\right]$

12. $\mathrm{C}_{\mathrm{n}}=\rho \mathrm{vl} \sqrt{ } \mathrm{n} / \pi+\rho \mathrm{vl} / \pi\left(\mathrm{n}-\sqrt{n}_{\mathrm{n}}\right) \alpha+\rho(1 / 4)^{2} \mathrm{n}(1-\alpha)\left(\lambda_{\mathrm{d}}-\lambda_{\mathrm{a}}\right)+\mathrm{d}_{\mathrm{L} 1 \mathrm{~L} 2}(\mathrm{n}-1) \rho(1 / 4)^{2} \mathrm{n}(1-\alpha) \lambda_{\mathrm{a}} / \mathrm{d}_{\mathrm{L} 1 \mathrm{Ln}}\left(\mathrm{R}_{\mathrm{c}} \mathrm{R}_{\mathrm{w}}+\right.$ $\mathrm{R}_{\mathrm{l}}$ )

13. $\mathrm{C}_{\mathrm{s}}=\mathrm{T}_{\mathrm{R}} * \mathrm{C}_{\mathrm{n}}$

14. $\mathrm{i} \leftarrow \mathrm{i}+1$

15. $\mathrm{CT}=\sum_{1}^{N} \mathrm{Cs}$ 
$\begin{array}{ll}\text { 16. } & \sum_{1}^{N}=\mathrm{N} \sum_{\mathrm{Cn}} * \sum_{\mathrm{Cs}}^{N} \\ \text { 17. } & \mathrm{ST}=\mathrm{TR} * \mathrm{PT} \\ \text { 18. } & \text { end while } \\ \text { 19. } & \text { end procedure }\end{array}$

\section{Performance Analysis}

The data association for signaling time looks for data that belongs to same cluster. It consists of detecting and associating noisy measurements, the origins of which may be unknown. The determination of signaling time is quite difficult in data fusion filtering. In this situations, either deterministic (based on Classical Hypothesis), or probabilistic (based on Bayesian Hypothesis) models are used. In this paper, the deterministic model has been used for the calculation. The values taken here are standard parameters [24]. It is found that the formulation of signaling time based on community model[1] yields better result than that of Waterfall Model, Multisensor Integration Fusion Model, Behavioural Knowledge-based Data Fusion Model, Omnibus Model, Dasarathy Model and JDL Model[10]-[14].

Table 2. The Standard Parameters

\begin{tabular}{ll}
\hline Parameter & Value \\
\hline Bias uncertainty $(\mathrm{o} / \mathrm{h})$ & $10-40$ \\
Scale factor stability $(\mathrm{ppm})$ & $100-500$ \\
Alignment $(\operatorname{arcs})$ & 200 \\
Random noise $(\mathrm{o} / \mathrm{h} / \sqrt{ } \mathrm{Hz})$ & $1-5$ \\
Flattening $(\mathrm{f})$ & $1 / 298.257223563$ \\
$\mathrm{R}_{\mathrm{w}}$ & 8 \\
$\mathrm{R}_{\mathrm{c}}$ & 0.5 \\
$\mathrm{R}_{\mathrm{l}}$ & 0.5 \\
$\mathrm{~d}_{\text {L1L2 }}$ & 16 \\
$\mathrm{~d}_{\text {L1Ln }}$ & $\sqrt{\mathrm{n}}$ \\
$\mathrm{V}$ & $28.9 \mathrm{~m} / \mathrm{s}$ \\
$\mathrm{P}$ & $0.0002 \mathrm{users} / \mathrm{m} 2$ \\
$\mathrm{~A}$ & 5 \\
$\lambda_{\mathrm{a}}$ & $0.0008 / \mathrm{s}$ \\
$\lambda_{\mathrm{d}}$ & $0.0008 / \mathrm{s}$ \\
\hline
\end{tabular}

Considering the number of LF levels as 1, the formulation of equation (3) yields $99 \mathrm{msg} / \mathrm{s}$ for community model and in case of other existing models, the values are 129, 117, 111, 118, 121 and $109 \mathrm{~s}$ for Waterfall Model, Multisensor Integration Fusion Model, Behavioural Knowledge-based Data Fusion Model, Omnibus Model, Dasarathy Model and JDL Model respectively and the comparison is shown in the form of bar chart in figure 8 .

Table 3. The Signalling Time

\begin{tabular}{ll}
\hline Model & Signaling Time \\
\hline Waterfall Model & 129 \\
Multisensor Integration Fusion Model & 117 \\
Behavioural Knowledge-based Data Fusion Model & 111 \\
Omnibus Model & 118 \\
Dasarathy Model & 121 \\
JDL Model & 109 \\
Community Model & 99 \\
\hline
\end{tabular}




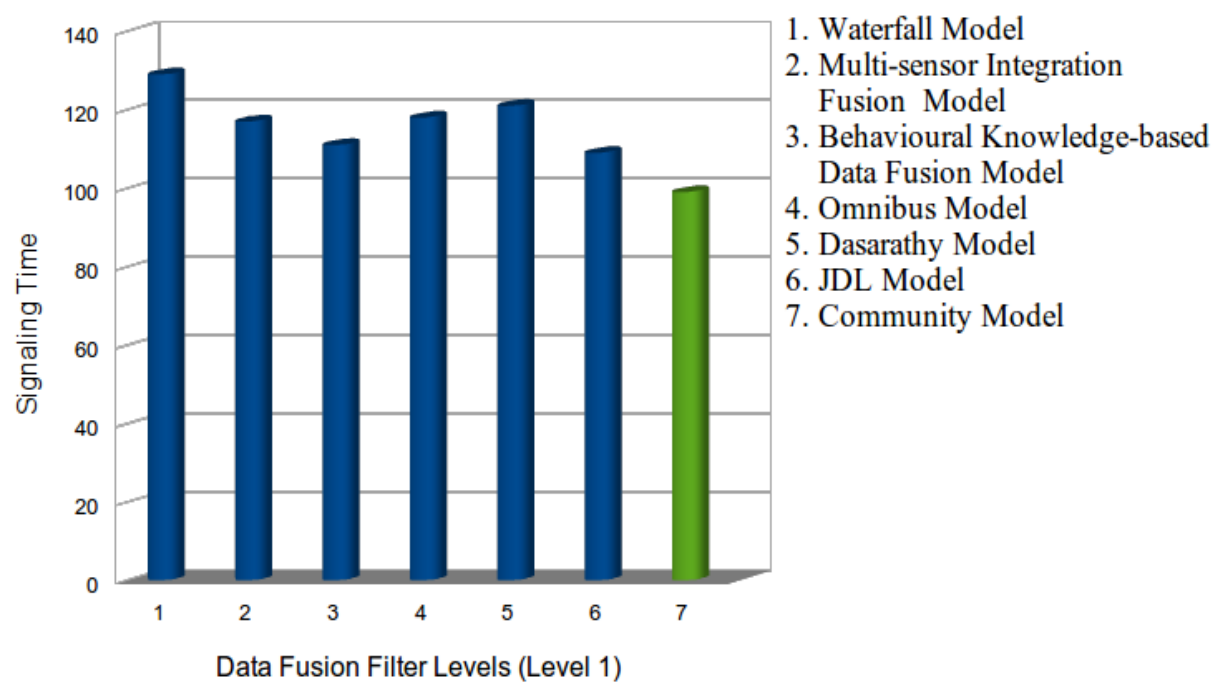

Fig. 8. Comparison of LF Signaling Time (Filter Level 1)

The above graph reveals that, the community model when compared to other existing models shows the least signaling cost irrespective of the filter levels. This happens due to the fact that, this model does not use the verification time as the filtered data are verified at each level of filter processing using the reference sensor. More over the data are fused only once after passing through all the filter levels.

\section{Conclusions}

This paper tries to put the new community model scheme to upgrade the signaling time in data fusion methodology. It also attempts to give the importance of the signaling time of the Community Model scheme of the data fusion in decision support system. The signaling time is an important parameter for data fusion as it determines the interrelationship among different signals to derive a proper relation among them. The performance evaluation based on the formulation of Signaling Time shows that the new Community Model is more efficient compared to other existing models. The behaviour of signaling time over different environments like cloud, bioinformatics, and quantum chromodynamics can be studied further to verify the effectiveness of these parameters.

\section{References}

[1] Bhattacharya, B., Saha, B. Community Model - A New Data Fusion Filter Paradigm, American Journal of Advanced Computing, Vol. II Issue I, January 2015, 25-31.

[2] Bhattacharya, B., Saha B. Community Model Architecture - A New Data Fusion Paradigm for Implementation, International Journal of Innovative Research in Computer and Commmucation Engineering, vol. 2, issue 6, (June 2014), 4774-4783.

[3] Bar-Shalom, Y., Fortmann T.E. Tracking and Data Association, Academic Press, San Diego, California, (1988).

[4] Bloch I., Maître H. Fusion de données en traitement d'images: modèles d'information et décisions, Traitement du Signal, vol. 11, no. 6, (1994), 435-446. 
[5] Blackman, S.S. Multiple Targets Tracking with Radar Applications. Artech House Inc. (1986)

[6] Hall, D. L., Llinas, J. An Introduction to Multisensor Data Fusion, Proceedings of the IEEE Vol. 85, No. 1, (Jan. 1997), 6 -23

[7] http://www.data-fusion.org/article.php (Last accessed on May 29, 2015)

[8] Luo, R. C., Kay, M. G. Multisensor Integration and Fusion in Intelligent System, IEEE Trans. on System, Man and Cybernetics, Vol. 19, No. 5, (Sept/Oct 1989), 901-931.

[9] Kokar, M., Kim, K. Review of Multisensor Data Fusion: Architecture and Techniques, Proceedings of The International Symposium on Intelligent Control, Chicago, Illinois, USA, (Aug. 1993), 261-266.

[10] Esteban, J., Starr, A., Willetts, R., Hannah, P., Bryanston-Cross P. A Review of Data Fusion Models and Paradigms: Towards Engineering Guidelines, Journal Neural Computing and Applications Volume 14 Issue 4, (December 2005) 273-281.

[11] Elmenreich, W. A Review on System Architectures for Sensor Fusion Applications, Lecture Notes in Computer Science, Springer, Vol. 4761, (2007) 547-559.

[12] Luo, R. C., Chang C. C., Lai, C. C. Multisensor Fusion and Integration: Theories, Applications, and its Perspectives, IEEE Sensors Journal, Vol. 11 No. 12, (December 2011), 3122-3138.

[13] Bedworth, M. D., O’Brien, J. C. The Omnibus Model: A New Model of Data Fusion?, Aerospace and Electronic Systems Magazine, IEEE, Vol. 15 Issue 4, (April 2000), 30-36.

[14] Nakamura, E. F., Loureiro A. A. F., Frery, A. C. Information Fusion for Wireless Sensor Networks: Methods, Models, and Classifications, ACM Computing Surveys, Vol. 39 No. 3, (August 2007), 9/1-9/55.

[15] Zhang, X., Castellanos, J. G., Campbell A. T. P-MIP: Paging Extension for Mobile IP Columbia University, (2002), 127-141.

[16] Wang, M., Georgiades, M., Tafazolli R. Signalling Cost Evaluation of Mobility Management Schemes for Different Core Network Architectural Arrangements in 3GPP LTE/SAE Vehicular Technology Conference 2008, (May 2008), 2253-2258.

[17] Karatsinides, S. E. Enhancing Filter Robustness in Cascaded GPS-INS Integrations, IEEE Transactions on Aerospace and Electronic Systems, Vol. 30, No. 4, (Oct., 1994), 1001-1008.

[18] Bell, W.B., Gorre, R.G., Cockrell, L.D. Cascading Filtered DTS Data into a Loosely Coupled GPS/INS System, Proceedings of IEEE PLANS'98, (1998), 586-593.

[19] Carlson, N.A. Federated Filter for Fault-Tolerant Integrated Systems, Proceedings of 1988 IEEE PLANS, (1988), 110-119.

[20] Felter, S.C. An overview of decentralized Kalman filter techniques, Proceedings of IEEE Southern Tier Technical Conference, (1990), 79-87.

[21] Liggins, M. E., Chong, C-Y., Kadar, I., Alford, M. G., Vannicola, V., Thomopoulos, S. Distributed Fusion Architectures and Algorithms for Target Tracking, Proceedings of the IEEE, Vol. 85, No. 1, (Jan. 1997), 95-107.

[22] Evans, F.A., Wilcox, J. C. Experimental Strapdown Redundant Sensor Inertial Navigation System, Journal of Spacecraft and Rockets, Vol. 7, No. 9, (Sept., 1970), 1070-1074.

[23] Potter, J.E., Deckert, J.C.: Minimax Failure Detection and Identification in Redundant Gyro and Accelerometer System, Journal of Spacecraft, Vol. 10, No. 4, (April, 1973), 236-243.

[24] Spitzer, C.R.: The Avionics Handbook, CRC Press LLC, (2001).

[25] Signalling Cost Analysis of Community Model, FICTA 2014, Bhubaneswar, India, Springer, Advances in Intelligent Systems and Computing, Vol 328 (2014), 49-56.

\section{Authors' Profiles}




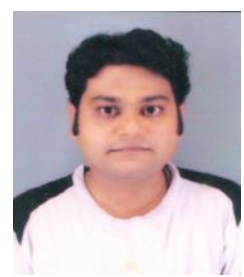

Boudhayan Bhattacharya is an assistant professor, Department of Computer Application in Sabita Devi Education Trust - Brainware Group of Institutions. He received his M. Tech (CSE) degree from West Bengal University of Technology, Kolkata, India. His research interests are Data Fusion, Mobile Computing, NoC etc.

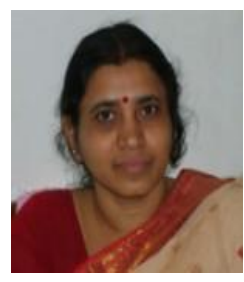

Dr. Banani Saha is an associate professor, Department of CSE in University of Calcutta. She received her $\mathrm{Ph}$. D (Tech) degree from University of Calcutta, India. Her research interests are Data Mining, Data Warehousing, Image Processing, Data Fusion etc.

How to cite this paper: Boudhayan Bhattacharya, Banani Saha,"Analysis of Signalling Time of Community Model", International Journal of Mathematical Sciences and Computing(IJMSC), Vol.1, No.2, pp.8-21, 2015.DOI: 10.5815/ijmsc.2015.02.02 